\title{
The necessity to use selective NADPH oxidase inhibitors
}

\author{
Shiho Satomi ${ }^{1} \cdot$ Shinji Kawahito $^{1} \cdot$ Hiroyuki Kinoshita $^{1,2}$ \\ Received: 11 August 2018 / Accepted: 8 September 2018 / Published online: 19 September 2018 \\ (c) Japanese Society of Anesthesiologists 2018
}

Keywords Apocynin · Diphenylene iodonium · NADPH oxidase

To the Editor:

Kito et al. [1] have reported that acute hyperglycemia impairs endothelium-dependent dilation of the rat pial arteriole. They also have quoted in their publication [1] that the NADPH oxidase inhibitors restore the vascular dysfunction. We have several concerns regarding their methodology, as well as the conclusion [1]. Both apocynin and diphenylene iodonium (DPI) are flavoprotein inhibitors, which play roles as non-selective inhibitors of reactive oxygen species, whereas Kito et al. adopted the agents as NADPH oxidase inhibitors in their study [1-3]. Indeed, previous studies proved that apocynin and DPI are non-selective antioxidants, and therefore, we should not use them as NADPH oxidase inhibitors in studies related to the vascular system [2, 3]. Therefore, we strongly recommend Kito et al. [1] to employ selective NADPH oxidase inhibitors, including gp91ds-tat to conclude that NADPH oxidase contributes to the endothelial dysfunction of the rat pial arteriole caused by acute hyperglycemia [4]. Collectively, we would like to await additional studies to verify the conclusion by Kito et al. [1].

This comment refers to the article available at https://doi. org/10.1007/s00540-018-2507-7.

An author's reply to this comment is available at https://doi. org/10.1007/s00540-018-2553-1.

Hiroyuki Kinoshita

hkinoshi@krc.biglobe.ne.jp

1 Department of Anesthesiology, Institute of Biomedical

Sciences, Tokushima University Graduate School, 3-18-15

Kuramoto, Tokushima, Tokushima 770-8503, Japan

2 Department of Anesthesiology, IMS Fujimi General Hospital, 1967-1 Tsuruma, Fujimi, Saitama 354-0021, Japan
Funding The institutional funding supported this study.

Conflict of interest Hiroyuki Kinoshita is a consult of IMI Co. Ltd, Koshigaya, Saitama, Japan.

\section{References}

1. Kito K, Tanabe K, Sakata K, Fukuoka N, Nagase K, Iida M, Iida $\mathrm{H}$. Endothelium-dependent vasodilation in the cerebral arterioles of rats deteriorates during acute hyperglycemia and then is restored by reducing the glucose level. J Anesth. 2018;32:531-8.

2. Heumüller S, Wind S, Barbosa-Sicard E, Schmidt HH, Busse $\mathrm{R}$, Schröder K, Brandes RP. Apocynin is not an inhibitor of vascular NADPH oxidases but an antioxidant. Hypertension. 2008;51:211-7.

3. Ratz JD, McGuire JJ, Anderson DJ, Bennett BM. Effects of the flavoprotein inhibitor, diphenyleneiodonium sulfate, on ex vivo organic nitrate tolerance in the rat. J Pharmacol Exp Ther. 2000;293:569-77.

4. Kinoshita H, Watanabe K, Azma T, Feng GG, Akahori T, Hayashi H, Sato M, Fujiwara Y, Wakatsuki A. Human serum albumin and oxidative stress in preeclamptic women and the mechanism of albumin for stress reduction. Heliyon. 2017;3:e00369. 